\title{
Kernos
}

Revue internationale et pluridisciplinaire de religion grecque antique

$14 \mid 2001$

Varia

\section{Un oracle médical de Sarpédon à Séleucie du Calycadnos}

\section{Cécile Nissen}

\section{OpenEdition Journals}

Édition électronique

URL : http://journals.openedition.org/kernos/774

DOI : $10.4000 /$ kernos.774

ISSN : 2034-7871

\section{Éditeur}

Centre international d'étude de la religion grecque antique

\section{Édition imprimée}

Date de publication : 1 janvier 2001

Pagination : 111-131

ISSN : 0776-3824

\section{Référence électronique}

Cécile Nissen, « Un oracle médical de Sarpédon à Séleucie du Calycadnos », Kernos [En ligne],

14 | 2001, mis en ligne le 14 avril 2011, consulté le 30 avril 2019. URL : http://journals.openedition.org/ kernos/774 ; DOl : 10.4000/kernos.774 


\section{Un oracle médical de Sarpédon à Sélleucie du Calycadnos*}

Pour tout historien du christianisme, la ville de Séleucie du Calycadnos évoque immanquablement le nom de sainte Thècle. Cette martyre possédait, dans la cité cilicienne, un sanctuaire réputé pour les miracles de guérison qu'elle y accomplissait. Par ailleurs, Séleucie est connue des historiens de l'Antiquité en tant que siège d'un oracle d'Apollon Sarpédonios. La confrontation des sources païennes et chrétiennes relatives à ces deux cultes apporte un éclairage nouveau sur ces dévotions. Déterminer les antécédents du culte de Thècle et préciser l'origine ainsi que la nature de la dévotion apollinienne, tel est le double objectif de cet article.

\section{Les sources païennes : l'oracle cilicien de Sarpédon}

L'implantation, en Cilicie, d'un centre oraculaire patronné par un Apollon qualifié de Sarpédonios ${ }^{1}$ est attestée à trois reprises par la littérature antique. Diodore de Sicile $^{2}$ est le premier auteur, dès le $\mathrm{I}^{\mathrm{er}}$ siècle av. J.-C., à faire mention d'un oracle « en Cilicie, à l'endroit où existe, dit-on, un sanctuaire d'Apollon Sarpédonios $»^{3}$. L'historien grec relate, dans un fragment conservé par Photios, la consultation accordée par cet oracle au roi de Coélé-Syrie et d'Antioche, Alexandre Balas, au milieu du II $^{\mathrm{e}}$ siècle av. J.-C. Apollon Sarpédonios lui conseilla de se méfier de l'endroit où était né l'être double; cet oracle énigmatique se révéla véridique lors de la mort du souverain à Abai, une cité d'Arabie qui avait vu naître un hermaphrodite.

* Cet article fait suite à un travail de fin d'études consacré aux sanctuaires médicaux en Asie Mineure. Cette enquête m'a permis de relever la présence en Anatolie de plusieurs cultes dont la vocation thérapeutique est aujourd'hui ignorée; l'oracle d'Apollon Sarpédonios à Séleucie du Calycadnos en fait partie. Je tiens à remercier R. Laffineur et V. Pirenne pour leur soutien et leurs conseils tout au long de l'élaboration de cet article.

1 L'oracle cilicien d'Apollon Sarpédonios a été étudié par A. Bouché-LeclercQ, Histoire de la divination dans l'Antiquité III, Paris, 1880, p. 257-258, 352-353; H.W. PARkE, The Oracles of Apollo in Asia Minor, Londres/Sydney/Dover, 1985, p. 194-196; T.S. MACKaY, "The Major Sanctuaries of Pamphylia and Cilicia", ANRW II, 18.3 (1990), p. 2110-2113.

2 Drod. SIC., XXXII, ap. Photios, Bibl., 244, 377a - 378b.

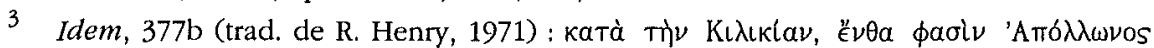

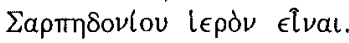


Quelque quatre siè̀cles plus tard, l'oracle cilicien d'Apollon Sarpédonios est toujours en activité. Zosime ${ }^{4}$ rapporte dans son Histoire nouvelle, la consultation oraculaire intervenue vers 270 ap. J.-C., soit peu avant l'expédition de l'empereur Aurélien contre les Palmyréens. Les sujets de la reine Zénobie, soucieux de savoir s'ils dirigeraient l'Orient, sont chassés du sanctuaire par le dieu, qui annonce, par ailleurs, la victoire d'Aurélien sur ses adversaires. L'apport le plus significatif du témoignage de Zosime réside dans la localisation du temple d'Apollon Sarpédonios à Séleucie de Cilicie ${ }^{5}$, c'est-à-dire à Séleucie du Calycadnos.

La Géographie de Strabon ${ }^{6}$ confirmait déjà l'établissement en Cilicie d'un sanctuaire oraculaire desservi par des prêtres inspirés. Cependant le témoignage de Strabon se démarque des deux précédents par l'attribution du temple et de son oracle à Artémis Sarpédonia ${ }^{7}$, et non à Apollon. Malgré cette divergence, il semble manifeste que le géographe désigne dans ce passage le même sanctuaire que Diodore, quelques années auparavant, et Zosime, environ cinq siècles plus tard. Localisation géographique, nature du culte et épiclèse de la divinité sont identiques; chaque auteur mentionne un sanctuaire oraculaire cilicien dédié à un dieu qualifié de Sarpédonien.

L'épithète appliquée à la divinité, qu'il s'agisse d'Apollon ou d'Artémis, évoque indéniablement le héros Sarpédon ${ }^{8}$. Allié des Troyens et chef du contingent lycien dans l'Iliade ${ }^{9}$, ce fils de Zeus et de Laodamie meurt sous les remparts de Troie, des œuvres de Patrocle. Son père veille alors au rapatriement de son corps par Hypnos et Thanatos, et à son inhumation dans sa Lycie natale ${ }^{10}$. Dans l'épopée homérique, Sarpédon apparaît donc surtout comme un héros lycien, fils de Zeus, deux caractères essentiels de son personnage qui lui sont conservés dans une deuxième tradition ${ }^{11}$.

\footnotetext{
4 Zos., I, 57.

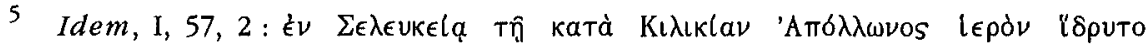

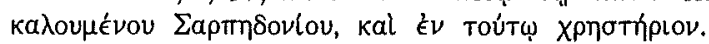

6 Strabon, XIV, 5, 19.

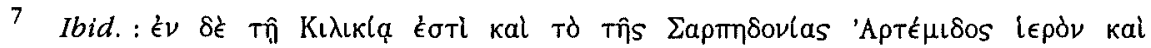

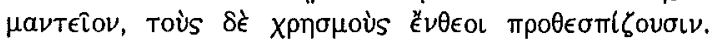

8 Sur la légende et le culte de Sarpédon, voir Roscher, s.v. Sarpedon (O. IMMisch), col. 400-411; RE, s.v. Sarpedon (J. ZWICKER, 1921), col. 36-43; LIMC, s.v. Sarpedon (D. voN Bothmer, 1994), p. 696-700; P. WATHELET, Dictionnaire des Troyens de l'Iliade II, Liège, 1988 (Documenta et Instrumenta, 1), p. 973-989.

9 Pour le rôle joué par Sarpédon dans l'épopée homérique : Hom., $I l$. VI, $198 s q_{\text {.; }}$ II, 876; V, $628 s q . ;$ XII, $101 s q ., 290 s q . ;$ XVI, $419 s q ., 666 s q$. La participation du fils de Zeus à la guerre de Troie est également mentionnée, à date beaucoup plus tardive, par Hygin (Fab., 106) et Apollodore (Epit. 3, 35; Epit. 4, 6).

10 Strabon $(X I I, 8,5)$ précise que, pour Homère, Sarpédon était né en Lycie même.

11 Cette deuxième version de la légende sarpédonienne qui met en scène un Sarpédon crétois - par opposition au premier que l'on peut qualifier de lycien - était déjà connue d'Hésiode (fr. 140 Merkelbach/West). Eschyle (Les Cariens ou Europe, fr. 99
} 
Il est, en effet, souvent présenté comme le fils de Zeus et d'Europe. Frère cadet de Minos et de Rhadamante ${ }^{12}$, il vit en Crète jusqu'à son départ pour l'Asie Mineure, conséquence d'une dispute avec Minos ${ }^{13}$. Il se réfugie en Carie où il fonde Milet $^{14}$ - dont la fondation est parfois attribuée à son compagnon Milétos $^{15}$-, puis devient roi de Lycie, après avoir soutenu son oncle maternel Cilix, roi de Cilicie, dans sa lutte contre les Lyciens ${ }^{16}$. Bien qu'elles considèrent toutes deux Sarpédon comme le roi des Lyciens, ces légendes sont incompatibles d'un point de vue chronologique; plusieurs générations séparent le règne de Minos de la guerre de Troie. C'est pourquoi certains auteurs accordent à notre héros une durée de vie exceptionnellement longue ${ }^{17}$, tandis que d'autres font du héros homérique le petit-fils du Sarpédon crétois ${ }^{18}$.

Les liens étroits tissés entre Sarpédon et la Lycie dans la mythologie s'accordent parfaitement avec la dévotion dont il faisait l'objet dans cette région méridionale de l'Anatolie ${ }^{19}$. Par contre, les récits mythiques traditionnels, véhiculés par la littérature païenne, ne signalent aucune liaison particulière entre Sarpédon et la Cilicie. Cette contrée limitrophe de la Lycie à l'est semble pourtant avoir réservé un accueil très favorable au fils de Zeus. L'ancrage cilicien de Sarpédon est reflété par des indices topographiques, mais aussi mythologiques. Ainsi, une troisième version de la légende sarpédonienne nous a été communiquée par un ouvrage chrétien du $\mathrm{v}^{\mathrm{e}}$ siècle ap. J.-

Nauck/Snell) et HÉrodote (I, 173) l'évoquent à leur tour. Elle apparaît encore à l'époque romaine, chez Strabon (XII, 8, 5; XIV, 1, 6; XIV, 3, 10) et Apollodore (III, 1, 1-2).

12 Aulu-Gelle $(X V, 21)$ fait de Sarpédon le frère de Minos et d'Eaque, et non de Rhadamante. Pour les variantes mineures de la généalogie de Sarpédon, voir WATHELET, o.c. (n. 8), p. 978.

13 Selon les auteurs, la querelle entre les deux frères concernait la royauté crétoise, ou l'amour du jeune Milétos qui partit avec Sarpédon.

14 Strabon, XII, 8, 5; Éphore, ap. Strabon, XIV, 1, 6. La nouvelle cité aurait été appelée Milet d'après le nom de l'établissement éponyme crétois d'où provenaient Sarpédon et ses compagnons.

15 D'après Apolıodore (III, 1, 2), Milétos fonda la cité à laquelle il donna son nom.

16 Apollod., III, 1, 2. - Hérodote (I, 173) et Strabon (XII, 8, 5; XIV, 3, 10) expliquent que l'actuelle Lycie s'appelait primitivement la Milyade et était peuplée par les Solymes; lors de la colonisation crétoise, les habitants prirent le nom de Termiles. C'est seulement plus tard, avec l'arrivée de Lycos, qu'ils furent appelés Lyciens. Sur la colonisation crétoise en Anatolie, voir Sp. Marinatos, "Les légendes royales de la Crète minoenne", Rev. Arch. 34 (1949), p. 12-17.

17 Apollodore (II, 1, 2) rapporte que Zeus accorda à son fils le privilège de vivre pendant trois générations.

18 Diod. Sic., V, 79, 3.

19 Un culte hérö̈que en l'honneur de Sarpédon était rendu en Lycie, notamment à

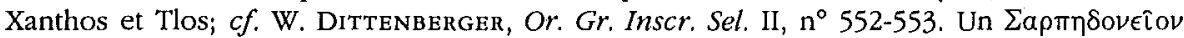
est même signalé à Xanthos par Schol. $I l$. (XVI, 673), et encore au $\mathrm{I}^{\text {er }}$ siècle av. J.-C. par Appien (Bell. civ., IV, 78). Pour le culte lycien de Sarpédon : Iмmisch, o.c. (n. 8), col. 407409; ZWICKER, o.c. (n. 8), col. 40-41. 
C., les Miracles de sainte Thècle, sur lequel je reviendrai plus longuement ciaprès $^{20}$. L'auteur de ce récit hagiographique rapporte l'histoire attachée au nom de Sarpédonios par les païens de Séleucie :

Il n'est personne qui ne connaisse notre Sarpèdonios : très ancienne est la légende qui le concerne et que nous ont apprise récits et livres. Certains savent même - parce qu'ils ont le tort d'être encore impies, mais comme on peut l'être de génération en génération - que cet homme fut jadis un étranger, venu loin de chez lui, errant à la recherche de sa soeur et déposé par la mer sur les côtes de ce pays; ils savent que, ne connaissant pas les lieux et ignorant qui régnait alors (c'était Kilix, son oncle, le frère de son père), il fut mis à mort pour avoir causé quelque dommage aux gens du cru et s'être attiré leur hostilité, et fut enterré sur cette avancée au bord de la mer. ${ }^{21}$

L'histoire contée dans ce passage rappelle étrangement l'épisode relaté par Apollodore au sujet de $\mathrm{Cilix}^{22}$, un des trois frères d'Europe partis à sa recherche après son enlèvement par Zeus. Leur père Agenor leur ayant interdit de rentrer en Phénicie sans leur sœur, les trois héros s'établirent à l'étranger; Cilix s'installa en Asie Mineure, dans la région qui prendra son nom. La légende du Sarpédonios, également appelé Sarpédon (les deux formes, $\Sigma a \rho \pi \eta \delta \delta$ vios et $\Sigma a \rho \pi \eta \delta \omega ́ v$ sont utilisées indifféremment dans le texte des Miracles $^{23}$ ) honoré à Séleucie, est construite sur le même schéma que celle de Cilix, du moins dans sa première partie. Certains identifient d'ailleurs le Sarpédon cilicien à un frère d'Europe.

Mais, à la différence du Cilix d'Apollodore, le Sarpédon honoré à Séleucie s'oppose aux indigènes anatoliens; il est tué par les sujets de son oncle ${ }^{24}$ et enterré en Cilicie. Cette version régionale du mythe de Sarpédon apparaît comme le reflet d'un culte local rendu à Sarpédon, en particulier à Séleucie ${ }^{25}$. Cette dévotion sarpédonienne devait prendre place autour d'un tombeau situé en bord de mer et identifié par la légende à la sépulture de l'étranger, qui, en échange des offrandes et des prières de la communauté, assurait la protection de ses membres.

Le lien entre Sarpédon et la Cilicie transparaît également dans la toponymie. Le promontoire sablonneux au large de Séleucie du Calycadnos, aujourd'hui dénommé Lisan-el-Kahbe, portait dans l'Antiquité, le nom de cap

20 L'apport des Miracles de sainte Thècle dans l'étude des cultes de Séleucie sera envisagé ci-dessous : infra, p. 119-122.

21 Miracles de sainte Thècle I, 1-10 (trad. de G. Dagron, 1978).

22 Cf. Apollod, III, 1, 1.

23 Infra, n. 35.

24 Dans la variante cilicienne de la légende, Cilix est présenté comme l'oncle paternel de Sarpédon, le frère de son père, dont l'identité n'est pas révélée, alors que, dans la version traditionnelle, Cilix est l'oncle maternel de Sarpédon.

25 Pour l'interprétation de cette légende séleucienne par l'auteur chrétien des Miracles : infra, p. 128. 
Sarpédon ${ }^{26}$. Formé par les alluvions du Calycadnos, ce cap étroit sur la côte

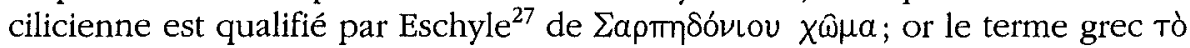
$\chi \hat{\omega} \mu \alpha$ peut désigner aussi bien une élévation de terre naturelle de type dune, promontoire, que l'amas de terre d'un tombeau, une tombe. Le vocabulaire choisi par le tragique entretient donc une incertitude dans l'interprétation de ce lieu : désigne-t-il un promontoire surnommé Sarpédonien ou la tombe de Sarpédon? On peut même imaginer que le promontoire était qualifié de Sarpédonien car il abritait le tombeau du héros. Cette hypothèse nous renvoie au témoignage de Strabon ${ }^{28}$ qui signale le cap Sarpédon formé par le

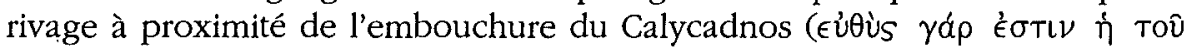

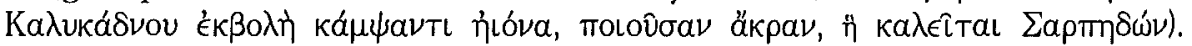

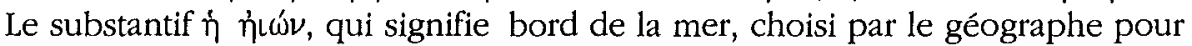
localiser le promontoire Sarpédon (í ăk $\rho \alpha$ ) est identique au terme utilisé dans les Miracles de sainte Thècle ${ }^{29}$ pour situer le tombeau de Sarpédon à

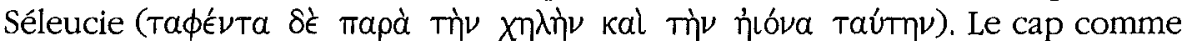
la tombe se trouvaient en bord de mer, peut-être au même endroit; la terminologie grecque soutient à nouveau la possibilité que la tombe de Sarpédon, siège de son culte, se soit élevée sur le promontoire dit Sarpédonien au sud de Séleucie.

$\mathrm{Au}$ vu de ces indications mythologiques et toponymiques, l'implantation de Sarpédon en Cilicie, plus particulièrement dans la région de Séleucie du Calycadnos, est évidente. Cependant, les textes relatifs au centre oraculaire cilicien attribuent l'oracle, non à Sarpédon, mais à Apollon ou à Artémis, tous

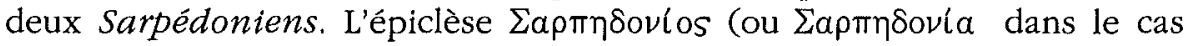
d'Artémis) trahit l'assimilation qui s'est produite entre Sarpédon et le fils de Léto. L'implantation du dieu de Delphes à Séleucie s'est produite au plus tard au I $^{\mathrm{er}}$ siècle av. J.-C. ${ }^{30}$, puisque Diodore de Sicile attribue déjà l'oracle cilicien à Apollon Sarpédonios. Ce dernier a repris à son compte un oracle plus ancien patronné par une figure locale dont il a conservé le souvenir dans son épiclèse. La substitution d'Apollon à Sarpédon semble, de prime abord, difficile à expliquer. Sur le plan mythologique, aucun épisode ne rapproche

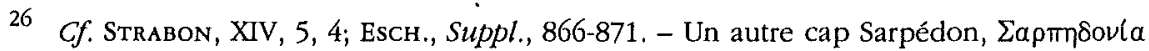
$a ̆ k \rho a$, est attesté en Thrace, à proximité de l'embouchure du fleuve Hébros, sur la côte face à Samothrace. Cf. Hdt, VII, 58; Strabon, VII, 331; Eudoxe de CNide, fr. 305 Lasserre; Schol. à APOLL. RH., I, 922 Teubner.

27

EscH., Suppl., 866-871.

28 Strabon, XIV, 5, 4.

29 Miracles de sainte Thècle I, 9-10.

30 La présence d'Apollon Sarpédonios à Séleucie est peut-être plus ancienne que le dernier siècle avant notre ère. La consultation relatée par Diodore (XXXII, ap. Photios, Bibl., 244, 377a-378b) remonte en effet au règne d'Alexandre Balas en Syrie, c'est-à-dire au milieu du II $^{\mathbf{e}}$ siècle av. J.-C. Il se peut cependant que l'historien ait décrit un événement passé en se fondant sur la réalité contemporaine. Comme Apollon patronnait l'oracle cilicien à son époque, Diodore aura pensé qu'il en allait déjà de même au siècle précédent. 
ces deux fils de Zeus. Apollon n'intervient dans la légende de Sarpédon qu'à la mort de celui-ci afin de nettoyer son corps, et ce, sur l'ordre de son illustre père ${ }^{31}$.

Néanmoins, quelques indices susceptibles d'expliquer l'institution du culte d'Apollon au détriment de Sarpédon doivent être signalés. La prise de pouvoir d'Apollon remonte peut-être à la domination des Séleucides, dont le dieu de Delphes était le patron et le protecteur. De plus, l'origine lycienne ainsi que l'ascendance - fils de Zeus - communes de ces deux figures doivent être soulignées. De telles similitudes ont pu favoriser leur rapprochement dans une contrée qui constituait, par ailleurs, un centre privilégié de la mantique apollinienne ${ }^{32}$. Il a donc pu sembler relativement naturel de confier l'oracle archaïque d'un Sarpédon local au dieu grec de la divination. Il faut d'ailleurs noter que, même après l'introduction d'Apollon, les pèlerins ont dû rester fidèles à l'antique propriétaire du lieu. "Peut-être Apollon passait-il pour être simplement le patron de l'oracle, dont Sarpédon continuait à être le gardien proprement dit. ${ }^{33}$ Malgré l'installation d'une figure chrétienne dans leur cité, les habitants de Séleucie continuaient, au v e siècle ap. J.-C., d'interroger Sarpédon ${ }^{34}$. Mais ce dernier était alors dénommé aussi bien $\Sigma a \rho \pi \eta \delta \omega ́ \nu$ que $\Sigma a \rho \pi \eta \delta$ óvıos, preuve de l'assimilation précédemment effectuée entre Sarpédon et Apollon; l'antique destinataire du culte était désigné par son propre nom ou par l'épiclèse de son successeur ${ }^{35}$.

Diodore de Sicile et Zosime, tout comme l'auteur de la Vie et des Miracles de sainte Thècle s'accordent donc sur l'identification de Sarpédon à Apollon dans le sanctuaire séleucien. Pourtant, Strabon ${ }^{36}$ mentionne un sanctuaire oraculaire d'Artémis Sarpédonia en Cilicie. L'attribution de l'oracle cilicien à la sœur d'Apollon a été considérée par A. Bouché-Leclercq ${ }^{37}$ comme le résultat d'une erreur du géographe, qui se serait trompé sur le destinataire du culte. Ch. Picard, puis H.W. Parke, plus récemment, soutiennent au contraire, que le témoignage de Strabon constitue le seul reflet de l'association cultuelle des deux enfants de Léto à Séleucie. Picard ${ }^{38}$ replace

31 Ном., $l l$. XVI, 666 sq.

32 Apollon rendait des oracles à Patara, Cyanea et Sura. Sur les oracles apolliniens de lycie et de Cilicie, voir Bouché-LeclercQ, o.c. (n. 1), p. 255-258; Parke, o.c. (n. 1), p. 185197.

33 E. Rohde, Psyché. Le culte de l'âme cbez les Grecs et leur croyance à l'immortalité, Paris, Payot, $1952^{10}$ [Fribourg, 1894], p. 155, n. 1.

34 Vie de sainte Thècle XXVII, 53-56; Miracles de sainte Thècle I; XI, 11-17; XVIII, 30-32; XI, 14-16, 29-31.

35 Cf. G. Dagron, Vie et Miracles de sainte Thècle. Texte grec, traduction et commentaire, Bruxelles, 1978 (Subsidia Hagiographica, 62), p. 86-87.

36 Strabon, XIV, 5, 19.

37 Bouché-LeclercQ, o.c. (n. 1), p. 258.

38 Ch. Picard, Éphèse et Claros. Recherches sur les sanctuaires et les cultes de l'Tonie du Nord, Paris, 1922, p. 397-398. 
cet oracle commun d'Apollon et d'Artémis dans le contexte général de la divination apollinienne en Asie Mineure :.l'Ionie avec les célèbres temenoi de Claros et de Didymes dédiés à Apollon ainsi que l'Artémision d'Éphèse, défendait la primauté de ses cultes face aux traditions de Lycie-Cilicie. L'Apollon de Patara, associé à celui de $\operatorname{Délos}^{39}$, apparaissait comme le principal rival des sanctuaires ioniens. L'opposition était telle « qu'en Cilicie, [...], une Artémis rendait elle-même des oracles, office dont il fut traditionnel, en Ionie, qu'elle se désintéressât au profit de son frère ${ }^{40}$. Parke ${ }^{41}$ partage l'opinion de Picard, estimant qu'Apollon et Artémis jouissaient, tous deux, de facultés prophétiques à Séleucie du Calycadnos. Un partage similaire des fonctions était opéré à Sidyma, localité lycienne dont une inscription du II $^{\mathrm{e}}$ siècle ap. J.-C. mentionne un prophète à vie d'Apollon et d'Artémis ${ }^{42}$.

L'association d'Artémis et d'Apollon dans l'oracle séleucien, autrefois patronné par Sarpédon, est confirmé par la numismatique ${ }^{43}$. En effet, dès le II $^{\mathrm{e}}$ siècle ou le $\mathrm{I}^{\mathrm{er}}$ siècle av. J.-C., la figure d'Artémis intervient, en buste, sur le monnayage en bronze de Séleucie ${ }^{44}$. En outre, des monnaies de l'époque d'Hadrien, présentent, au revers, les bustes affrontés d'Apollon et d'Artémis ${ }^{45}$. Ces deux types monétaires semblent donc corroborer, tout au moins, l'implantation du culte d'Artémis à Séleucie, et peut-être même une éventuelle fonction prophétique de la déesse de la chasse, vu son association avec Apollon.

L'étude des monnaies de Séleucie confirme aussi l'importance revêtue par le culte et l'oracle d'Apollon dans cette cité. En effet, outre la représentation de la tête du dieu à l'avers de quelques monnaies du $\mathrm{II}^{\mathrm{e}}$ siècle ou du $\mathrm{I}^{\mathrm{er}}$ siècle avant J.-C. ${ }^{46}$, Apollon est également figuré, face à Tyché, sur plusieurs types impériaux $^{47}$ frappés entre Gordien III et Valérien $\mathrm{I}^{\mathrm{er}}$. Or, sur ces dernières monnaies, le dieu est accompagné de la traditionnelle branche de laurier, son attribut le plus significatif en tant que dieu de la divination. Des exemplaires émis sous Marc Aurèle, portent d'ailleurs, au revers, cette seule branche de laurier ${ }^{48}$.

39 L'Apollon délien était réputé passer l'hiver à Patara. Cf. VIRG., Enéide IV, 143-145.

40 Picard, o.c. (n. 38), p. 398.

41 PARKe, o.c. (n. 1), p. 194, 196.

42 IGRRP III, 583. Cf. PARKe, o.c. (n. 1), p. 191.

43 L'apport de la numismatique dans l'étude des cultes de Séleucie a été montré par Mackay, o.c. (n. 1), p. 2112.

44 BMC Lycaonia, p. 130, $\mathrm{n}^{\circ} 15$.

45 Idem, p. $131, \mathrm{n}^{\circ} 17$.

46 Idem, p. $130, \mathrm{n}^{\circ} 11-14$.

47 Idem, p. 139-141, n' 47-53, 55.

48 Idem, p. $132, \mathrm{n}^{\circ} 21-22$. 
Enfin, la numismatique nous renseigne sur l'origine du culte d'Apollon Sarpédonios à Séleucie. Strabon ${ }^{49}$ affirme, en effet, que lors de la fondation de Séleucie, Séleucos $I^{\text {er }}$ y transplanta la population de la localité voisine d'Holmi. Or, le principal type monétaire de cette cité représente Athéna au droit, associée à Apollon au revers, avec une phiale et une branche de laurier ${ }^{50}$. C'est pourquoi, G.F. Hill ${ }^{51}$ pense que les divinités vénérées à Holmi, en l'occurrence Apollon et Athéna ${ }^{52}$, ont également été transférés à Séleucie. Lors de leur arrivée à Séleucie, ces cultes nouveaux ont dû se superposer à des dévotions locales primitives; ainsi, il est probable que Sarpédon était déjà vénéré sur ce site et qu'il a été identifié au nouvel arrivant, Apollon, dont il partageait les compétences prophétiques.

\section{Les sources chrétiennes : la légende hagiographique de sainte Thècle}

La documentation littéraire païenne nous informe donc de l'implantation, en Cilicie, plus précisément à Séleucie du Calycadnos, d'un culte en l'honneur de Sarpédon, supplanté par Apollon Sarpédonios ou Artémis Sarpédonia. Plus qu'un simple culte local, les textes attestent la vocation oraculaire du sanctuaire. Or, la nature de cet oracle peut être précisée par des sources chrétiennes; plusieurs textes hagiographiques relatifs à sainte Thècle révèlent les compétences médicales attribuées à Sarpédon, et endossées par la sainte chrétienne lors de son arrivée à Séleucie.

Sainte Thècle ${ }^{53}$ est une célèbre figure du christianisme naissant : la formidable expansion de sa renommée est perceptible dans l'enrichissement progressif de sa légende au fil des siècles. Entre les Actes apocryphes de Paul et de Thècle ${ }^{54}$ rédigés au plus tard à la fin du $\mathrm{II}^{\mathrm{e}}$ siècle ap. J.-C. et la Vie et les Miracles de sainte Thècle ${ }^{55}$ datés du ve siècle ap. J.-C., la martyre est parvenue à se dégager de l'emprise de saint Paul pour acquérir une personnalité tout à fait indépendante, mise en scène dans des épisodes distincts. Sa

49 Strabon, XIV, $5,4$.

50 BMC Lycaonia, p. $85, \mathrm{n}^{\circ} 1$, p. L-LI.

51 Idem, p. LI, LXIV.

52 Pour le culte d'Athéna honorée sur l'acropole de Séleucie, voir T.B. Mitrond, "The Cults of Roman Rough Cilicia", ANRW II, 18.3 (1990), p. 2148. - Pour Athéna sur les monnaies de Séleucie : BMC Lycaonia, p. 128-142.

53 Sur sainte Thècle et son culte à Séleucie du Calycadnos, voir Dagron, o.c. (n. 35); H. Delehaye, "Les recueils antiques de Miracles de saints", Analecta Bollandiana 43 (1925), p. 49-57; A.J. Festugière, Sainte Thècle, saints Côme et Damien, saints Cyr et Jean (Extraits), saint Georges, Paris, éditions Picard, 1971, p. 11-82.

54 Ces Actes apocrypbes de Paul et de Thècle ont été publiés en français par L. Vouaux, Les Actes de Paul et ses lettres apocryphes, Paris, 1913.

55 Une traduction française et un nouveau commentaire de la Vie et des Miracles de sainte Thècle ont été proposés par Dagron, o.c. (n. 35). 
légende apparaît comme le résultat d'une élaboration littéraire très intense entre le $\mathrm{II}^{\mathrm{e}}$ siècle et le $\mathrm{v}^{\mathrm{e}}$ siècle ap. J.-C. L'histoire de Thècle, telle qu'elle est racontée par les manuscrits les plus récents des Actes de Paul ainsi que par la Vie de sainte Thècle, nous emmène d'Iconium, en Isaurie, où la jeune fille se convertit à l'écoute de la prédication de Paul, jusqu'à Séleucie du Calycadnos, en Cilicie Trachée, où elle accomplit de nombreux miracles de son vivant, mais également après sa mort; son tombeau devient, en effet, un lieu de pèlerinage très réputé, centre d'une véritable cité-sainte édifiée en son honneur ${ }^{56}$

La Vie et les Miracles de sainte Thècle indiquent comment la martyre s'est ịmposée dans la cité cilicienne au détriment d'un $\delta a i ́ \mu \omega \nu$ païen du nom

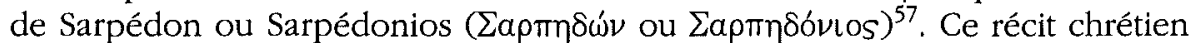
confirme donc la présence, à Séleucie, d'un homonyme du héros lycien ${ }^{58}$, qui n'est d'ailleurs pas le seul occupant paien auquel Thècle se heurte lors de son arrivée. Athéna, Aphrodite et Zeus sont également honorés par les indigènes. D'après l'auteur anonyme $e^{59}$ de ce double recueil hagiographique, la première initiative de Thècle consiste à évincer ces rivaux directs ${ }^{60}$. Pourtant, l'un d'entre eux n'abandonne pas totalement la place et continue de jouir d'un grand crédit auprès des habitants; il s'agit précisément de Sarpédon. Bien que Thècle l'ait réduit au silence dès le premier miracle ${ }^{61}$, comme l'affirme l'auteur des $\Theta a u ́ f u t a$, son nom est encore cité à trois reprises dans le reste du texte ${ }^{62}$.

Ces trois passages présentent une évidente similitude : à chaque fois, Sarpédon est consulté sur des questions d'ordre médical par des fidèles qui lui sont restés attachés. Un des miracles rapporte l'histoire d'un garçonnet du

56 Vie de sainte Thècle XXVIII, 15-17.

57 Pour l'explication de la double dénomination du héros dans cet ouvrage hagiographique : supra, n. 35. - La lutte entre Thècle et Sarpédon-Sarpédonios évoquée une première fois dans la Vie (XXVII, 53-56), est décrite plus longuement dans les Miracles (I).

58 Pour la légende de ce Sarpédon cilicien : supra, p. 113-114.

59 La Vie et les Miracles de sainte Thècle ont longtemps été attribués à Basile de Séleucie, évêque de la cité cilicienne au $\mathrm{v}^{\mathbf{e}}$ siècle ap. J.-C. Les manuscrits les plus anciens reproduisant cet ouvrage le désignent déjà comme l'œuvre de cet écrivain prolixe auteur de nombreuses homélies. Une critique interne sérieuse a, néanmoins, permis à Dagron de relever plusieurs indices convergents qui démontrent l'inexactitude de cette attribution. A plusieurs reprises, l'auteur de la Vie et des Miracles rappelle les différends qui l'ont opposé au clergé de Séleucie, notamment à Basile, alors évêque. Bien que son identité demeure inconnue, les quelques éléments autobiographiques disséminés au long du texte révèlent que cet hagiographe devait être un ancien rhéteur originaire de Séleucie, devenu prêtre lorsqu'il rédigea la légende de Thècle. $C f$. DAGron, o.c. (n. 35), p. 13-30.

60 Pour la rivalité entre Thècle et ses prédécesseurs païens : Vie de sainte Thècle XXVII, 53-62; Miracles de sainte Thècle, I-IV.

61 Miracles de sainte Thècle I, 15-20.

62 Idem XI, 11-17; XVIII, 30-32; XI, 14-16, 29-31. 
nom d'Aurélios ${ }^{63}$, souffrant d'écrouelles ${ }^{64}$. Devant l'impuissance des médecins, " la grand-mère de l'enfant implora le très cher Sarpédonios, grand médecin à ce qu'on dit, comme peut invoquer un démon une femme dont l'esprit est égaré par les démons, et lui non plus ne sut indiquer le moyen de guérir, soit qu'il se fût tout à fait tu, soit que (comme c'est son habitude) il eût trompé la femme et l'eût renvoyée sans profit après avoir proféré une énigme, une fable, ou n'avoir pas du tout ouvert la bouche ${ }^{65}$. C'est alors qu'intervient Thècle; elle apparaît à la vieille femme et lui révèle le remède qui guérira son petit-fils.

Cet épisode s'inscrit manifestement dans le cadre d'une propagande chrétienne, visant à affirmer la suprématie de la martyre sur tous ses concurrents. Thècle réussit là où médecins et démons paỉens avaient échoué. Même si la volonté de l'auteur était de souligner le pouvoir de la sainte et, en corrélation, l'impuissance de Sarpédon, il témoigne des compétences médicales reconnues au $\delta a l \mu \omega \nu$ local par les autochtones. La grand-mère de l'enfant malade se tourne spontanément vers Sarpédon, dont le texte des Miracles

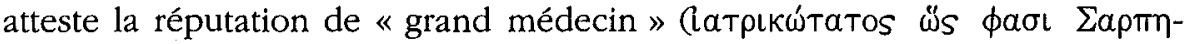
sóvios) parmi la population.

Sarpédonios est à nouveau mentionné lors du récit d'une autre guérison miraculeuse ${ }^{66}$ accordée par Thècle. Aba, une païenne originaire de Séleucie, souffrait de la jambe à la suite d'une chute de cheval. Son mal empire avec le temps, si bien que «la femme ne pouvait plus bouger, malgré ceux qui se jouaient d'elle, tantôt les juifs, tantôt nos faiseurs d'incantations et avec eux l'excellent Sarpédonios, qui prétendaient la soigner ou même qui faisaient effectivement quelque chose, mais sans pouvoir et, en fin de compte, sans avoir pu arriver à rien ${ }^{67}$. La malade se rend alors au « temple » de Thècle, qu'elle quitte guérie, et convertie, trois jours plus tard. Ce récit démontre la

63 Idem XI.

64 Bien que le terme d'écrouelles soit aujourd'hui vieilli, il désigne, dans ce cas, une

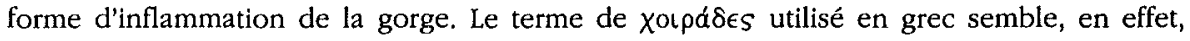
correspondre à une affection symptomatique de la tuberculose ganglionnaire. Cette dernière pouvait être accompagnée d'une inflammation de la gorge, des oreilles, puis de la tuméfaction des glandes au cou, aux aisselles et aux aines; ce sont ces diverses mani-

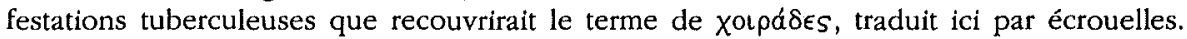
Cf. M. Grmek, Les maladies à l'aube de la civilisation occidentale, Paris, 1994, p. 289-290.

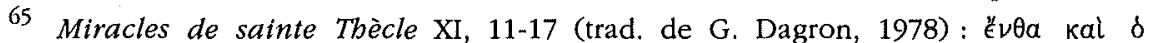

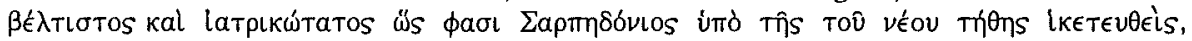

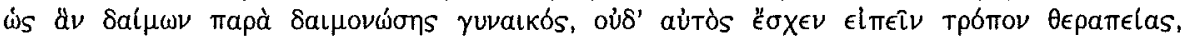

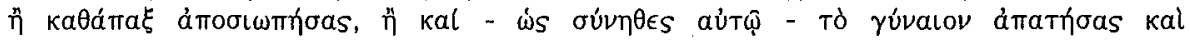

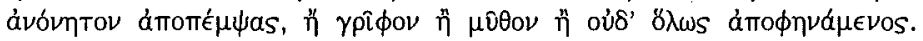

66 Idem XVIII.

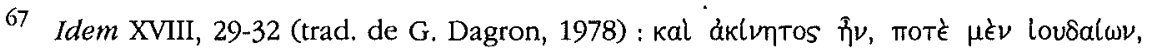

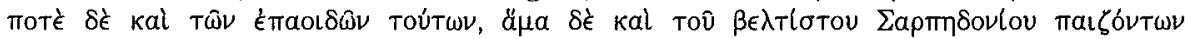

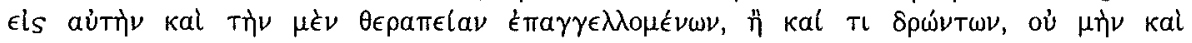

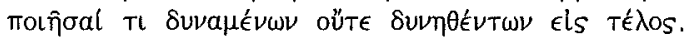


similitude des fonctions attribuées à Thècle et Sarpédon. L'un et l'autre apparaissent comme des figures guérisseuses, même si, dans ce cas comme dans le précédent, l'auteur cherche à prouver l'inefficacité du $\delta a l \mu \omega \nu$ païen face à la puissance de la sainte.

Un dernier miracle de Thècle $^{68}$ témoigne du rôle médical accordé à Sarpédon à Séleucie. La martyre indique au rhéteur Arétarchos, atteint d'une maladie des reins, le remède de son mal. Mais, après sa guérison, l'homme demeure païen, puisque « c'est à un autre [que Thècle] qu'il attribue la grâce de la guérison : "C'est Sarpédonios - dit-il - qui m'a prescrit d'aller le [le remède] demander à la martyre et de le prendre." " ${ }^{69}$ Outre la pérennité de la dévotion à Sarpédon, ce miracle fournit une indication importante relative à l'histoire du culte de Séleucie. L'hagiographe, s'élevant contre l'aveuglement d'Arétarchos, proclame la puissance de Thècle : « Ah, puisse ton âme être guérie par la martyre, même si tu devais en attribuer la responsabilité à Sarpédon, à Apollon, ou au démon que tu voudras. ${ }^{70}$ Cet extrait confirme l'implantation successive, ou concomitante, à Séleucie, de Sarpédon et d'Apollon. Elle apporte la preuve que l'épiclèse Sarpédonios portée par Apollon dans cette cité renvoie à un autre culte séleucien, vraisemblablement plus ancien, en l'occurrence un culte en l'honneur de Sarpédon.

Les histoires d'Aurélios, d'Aba et d'Arétarchos démontrent combien la confiance en les pouvoirs thérapeutiques de Sarpédon était forte parmi les habitants. Or, si les qualités de guérisseuse de Thècle sont aujourd'hui communément admises $^{71}$, le statut de médecin n'est jamais reconnu à son adversaire païen. Les Actes de Paul ainsi que la Vie et les Miracles de Thècle affirment, à diverses reprises, combien la sainte était vénérée pour ses facultés thérapeutiques. Dès son installation dans une grotte près de Séleucie, elle opère nombre de guérisons si bien que les fidèles en proie à la maladie affluent ${ }^{72}$. Ce sont d'ailleurs les médecins de Séleucie, auxquels elle porte ombrage, qui provoquent sa disparition sous terre; ils lui envoient des jeunes gens chargés de la corrompre afin de lui retirer ses dons de thérapeute $^{73}$. Mais après sa « mort », sa renommée ne cesse de s'amplifier et elle

68 Idem XI.

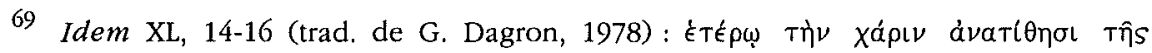

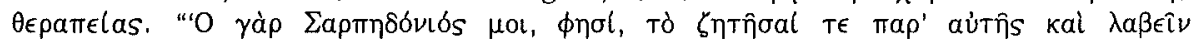

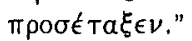

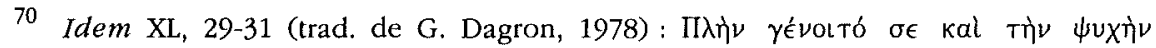

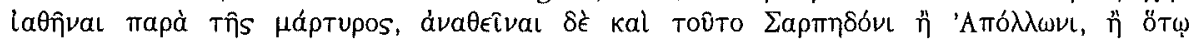

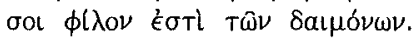

71 Cf. Dagron, o.c. (n. 35), p. 101-108.

72 Les manuscrits grecs A, B, C, et surtout $\mathrm{G}$ et $\mathrm{M}$, des Actes de Paul et de Thècle, XLIV, décrivent, de manière plus ou moins détaillée, l'installation de Thècle à Séleucie et son activité de guérisseuse.

73 Sur la fin de Thècle et l'histoire des médecins, voir Actes de Paul et de Tbècle XLIV, XLV. 
accorde encore d'innombrables guérisons; sur la cinquantaine de miracles relatés par l'auteur des Өaú $\mu a t a$, un tiers concernent des malades qui recouvrent la santé sur les conseils de la martyre ${ }^{74}$.

Les miracles opérés en faveur d'Aurélios, d'Aba et d'Arétarchos s'intègrent donc parfaitement au portrait de Thècle dressé par la légende. Ils mettent en scène une sainte bienveillante dotée de pouvoirs guérisseurs sans limite. Ces trois récits apportent cependant une précision décisive : au v $\mathrm{v}^{\mathrm{e}}$ siècle ap. J.-C., Sarpédon n'avait pas été totalement supplanté par son adversaire chrétienne. Si la martyre avait acquis une domination sans partage sur les cultes de Séleucie, pourquoi notre hagiographe prendrait-il encore la peine de rappeler la supétiorité de Thècle à travers ces trois exemples? Et si la renommée de Thècle avait été aussi considérable que les auteurs chrétiens l'affirment, pourquoi trois malades s'adresseraient-ils encore à Sarpédonios? La sainte aurait dû leur apparâtre comme l'unique recours susceptible de leur rendre la santé, comme le prouvent d'ailleurs les trois dénouements. C'est que Sarpédonios avait conservé, malgré la célébrité nouvelle de Thècle, un certain prestige. Arétarchos nous en donne la preuve : bien que la martyre lui procure le remède approprié à ses maux, il demeure persuadé qu'il doit la guérison à Sarpédonios. L'ancienneté même du culte sarpédonien devait apparaître, aux yeux d'une partie du peuple, comme un gage de sa puissance; Sarpédon était le $\delta \alpha\lfloor\mu \omega \nu$ du lieu, honoré depuis toujours, celui auquel les Séleuciens avaient de tout temps adressé leurs prières.

Mais quelles étaient ces prières ? Zosime, et surtout Diodore, s'ils confirment l'antiquité de l'oracle d'Apollon Sarpédonios, et à plus forte raison celle de son prédécesseur Sarpédon, ne lui confèrent pas de statut particulier. La nature des questions posées au dieu paraît tout à fait ordinaire; dans les deux cas, les consultations portent sur des problèmes d'ordre politique. Les Miracles de Thècle offrent, à ce propos, un éclairage nouveau : lors de ses trois apparitions, le $\delta a i ́ \mu \omega \nu$ païen est présenté comme le rival de Thècle dans le domaine médical. Sarpédon était, comme. Thècle à sa suite, un guérisseur. Les histoires d'Aurélios, d'Aba et d'Arétarchos reflètent la nature primitive du culte sarpédonien de Séleucie: il s'agissait d'un culte thérapeutique. La réputation de ce Sarpédon médecin devait être telle que la dévotion chrétienne ne s'est imposée que difficilement au terme de plusieurs siècles de concurrence. Thècle a été obligée, pour se substituer à son prédécesseur, d'assumer les fonctions médicales qu'il remplissait.

Conformément aux pratiques en usage dans la plupart des sanctuaires

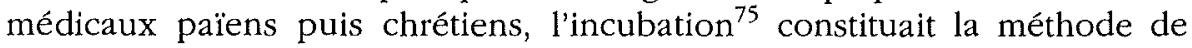

74 Miracles de sainte Thècle VII, VIII, XI, XII, XIV, XVII-XIX, XXIII-XXV, XXXVI-XLII,

75 Sur le recours à l'incubation dans les sanctuaires médicaux de l'Antiquité, voir $R E$, s.v. Incubatio (Pley, 1916), col. 1256-1262; $O C D^{3}$, s. v. Incubation, p. 753-754; A. BouCHÉLeclercQ, Histoire de la divination dans l'Antiquité I, Paris, 1879, p. 273-329; C.A. MeIER, "Le rêve et l'incubation dans l'ancienne Grèce", in R. Calllois et G.E. von Grüngbaum (éds), Le rêve et les sociétés bumaines, Paris, Gallimard, 1967, p. 290-305. - La pratique de 
consultation privilégiée à Séleucie, dans le sanctuaire de Thècle ${ }^{76}$. La sainte apparaît en songe au malade endormi qui implore son intervention et elle lui indique un remède. Comme pour les guérisons divines opérées durant l'Antiquité tardive ${ }^{77}$, l'incubation ne se déroule pas nécessairement dans le sanctuaire, mais peut se produire en n'importe quel endroit, notamment chez le patient ${ }^{78}$. Mais, à la différence de l'incubation païenne, Thècle offre fréquemment son secours à des patients qui n'ont pas sollicité son aide. Les consultations accordées par la martyre relèvent donc plutôt de l'oniromancie au sens large que de l'incubation proprement dite $e^{79}$.

Or, Tertullien affirme, dans son traité De anima, que Sarpédon possédait, en Troade, un oracle où était pratiquée la divination par les rêves ${ }^{80}$. Cette

l'incubation thérapeutique a été récupérée par le christianisme; outre sainte Thècle, plusieurs saints guérisseurs ont repris à leur compte les procédures d'incubation antiques; saint Michel, saint Côme et saint Damien indiquent en rêve, aux croyants endormis dans leurs églises, le traitement qui leur apportera la guérison. $C f$. M. Delcourt, Les grands sanctuaires de la Grèce, Paris, 1947, p. 112-113. - De plus, l'incubation s'est perpétuée, dans la Grèce moderne, jusqu'au milieu de ce siècle : plusieurs églises orthodoxes recevaient encore des fidèles malades qui y passaient la nuit, dans l'espoir de se réveiller guéris le matin. Cf. J.C. Lawson, Modern Greek Folklore and Ancient Greek Religion. A Study in Survivals, New York, 1964, p. 61-63.

76 L'auteur des Miracles affirme que Thècle avait pour habitude d'apparaître aux

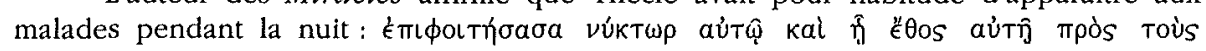

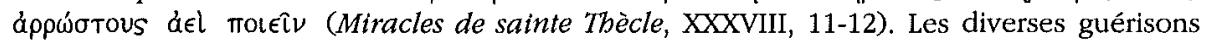
miraculeuses qu'il expose, dans son ouvrage, illustrent d'ailleurs parfaitement son propos. Elles se déroulent toutes selon un même schéma: Thècle apparaît durant le sommeil du malade et lui révèle un traitement; à son réveil, le fidèle applique ce remède, souvent surprenant, et recouvre la santé. Cf. DaGron, o.c. (n. 35), p. 103-108; Delehaye, o.c. (n. 52), p. 52-53. - Séleucie n'était pas la seule cité cilicienne, où Thècle était vénérée; la sainte possédait, à Aigai, un second sanctuaire, où l'incubation était également pratiquée. Les Miracles de sainte Thècle XXXIX, relatent l'histoire du rhéteur Isokasios guéri après avoir dormi dans l'église de Thècle à Aigai.

77 Il ressort des témoignages littéraires et épigraphiques qu’à l'époque romaine, l'incubation ne se déroule plus nécessairement dans le sanctuaire du dieu consulté. Le patient peut recevoir un rêve thérapeutique aussi bien à son domicile qu'au cours d'un voyage. Le lieu n'est plus considéré comme un facteur déterminant de la consultation oniromantique. Cf. Th. LeFORT, "Notes sur le culte d'Asklépios, Nature de l'incubation dans ce culte, II. Époque romaine"; Musée belge 10 (1906), p. 107-109.

78 Deux miracles se produisent directement au domicile des malades (Miracles de sainte Thècle XI, XIV), tandis qu'une patiente originaire de Tarse reçoit la visite nocturne de la sainte lors d'une étape de son voyage vers Séleucie (idem XVIII).

79 Il faut signaler qu'à Séleucie, l'oniromancie n'était pas réservée aux guérisons. Elle pouvait aussi intervenir dans d'autres miracles. Par exemple, Thècle indique, en songe, à une jeune mariée, l'endroit où se trouve la ceinture qui lui a été volée (Miracles de sainte Thècle XXI).

80 Tertullien, De anima, 46, 11 : Ceterum Epicharmus etiam summum apicem inter divinationes somniis extulit cum Philochoro Atheniensi. Nam et oraculis boc genus stipatus est orbis, ut Amphiarai apud Oropum, Ampbilocbi apud Mallum, Sarpedonis in 
information de l'apologiste africain n'est cependant corroborée par aucun autre témoignage. C'est pourquoi elle a souvent été mise en doute ${ }^{81}$, d'autant qu'elle intervient dans une liste d'oracles oniromantiques choisis par l'auteur, à titre d'exemples, afin d'illustrer le succès de cette pratique à son époque. Il est possible que Tertullien ou la source sur laquelle ce dernier se fondait, ait commis une erreur sur la localisation de ce centre oraculaire. Peut-être évoque-t-il, dans ce passage, le culte sarpédonien de Séleucie du Calycadnos, qui demeure, par ailleurs, l'unique oracle en lien avec Sarpédon connu dans le reste de la documentation. Il est intéressant de noter que, dans cette série d'oracles, celui de Sarpédon est cité directement après celui d'Amphilochos près de Mallos, un oracle cilicien, tout comme celui de Sarpédon à Séleucie.

Quelle que soit la localisation exacte de cet oracle de Sarpédon, le témoignage de Tertullien nous informe du recours à l'oniromancie dans le culte sarpédonien. La divination par les rêves est un procédé fermement attesté chez la plupart des autres héros mentionnés par l'apologiste ${ }^{82}$. Or, à partir de l'époque hellénistique, et plus encore à l'époque romaine, l'oniromancie est devenue la méthode par excellence de la divination médicale. Si Sarpédon accordait des révélations en rêves, il est très probable qu'il était lui aussi consulté pour des problèmes de santé. Même si le propos de Tertullien ne concerne pas l'oracle de Séleucie, il évoque l'utilisation de la divination par les songes dans le culte de Sarpédon et confirme ainsi indirectement les compétences thérapeutiques du héros.

La procédure même de consultation de l'oracle sarpédonien a donc dû se transmettre à la dévotion chrétienne. Thècle a naturellement repris à son compte la méthode de l'incubation pratiquée par son prédécesseur, d'autant que cette technique était la plus courante dans les cultes médicaux de l'époque. Néanmoins, l'incubation a connu une évolution sensible dans le culte de Thècle. Elle était exercée aussi bien dans le sanctuaire qu'au-dehors, au profit de fidèles en proie à des problèmes variés, médicaux ou autres, que ces fidèles aient réclamé ou non l'assistance de la martyre. L'arrivée de Thècle a ainsi entraîné une transformation des pratiques cultuelles : l'incubation médicale a cédé la place à une forme d'oniromancie généralisée à des consultations de nature variée.

Troade, Trophonii in Boeotia, Mopsi in Cilicia, Hermionae in Macedonia, Pasiphaae in Laconica.

81 Pour Bouché-Leclerce (o.c. [n, 1], p. 352-353) et Rohde (o.c. [n. 30], p. 155, n. 1), cet oracle de Sarpédon en Troade est le résultat d'une erreur de Tertullien. - Par contre, Immisch (o.c. [n. 6], col. 400) défend l'hypothèse selon laquelle, lors de la guerre de Troie, Sarpédon a été inhumé en Troade même où son tombeau est devenu le centre d'un culte héroïque.

82 Il ne fait aucun doute que l'oniromancie était pratiquée dans les sanctuaires d'Amphiaraos, Trophonios et Pasiphaé. 


\section{Pérennité de la nature médicale du culte de Séleucie de Calycadnos}

La confrontation des témoignages littéraires païens et chrétiens donne une image beaucoup plus précise de la dévotion sarpédonienne de Séleucie du Calycadnos. La nature oraculaire du sanctuaire cilicien, le remplacement du $\delta a i \mu \omega \nu$ par une sainte guérisseuse, la réputation de médecin conservée par Sarpédon chez les païens ainsi que la pratique de l'incubation dans le culte sarpédonien, tous ces indices permettent d'affirmer que le sanctuaire de Séleucie abritait, à l'origine, un culte thérapeutique patronné par Sarpédon. Par sa vocation médicale, ce culte occupait une position centrale dans les croyances des habitants. Il intervenait dans leur vie quotidienne et répondait à des préoccupations fondamentales liées à leur santé physique. Sa suppression était, dès lors, impossible, à moins d'offrir une contrepartie aux citoyens. C'est pourquoi, le nouveau culte, dédié à sainte Thècle, a dû pour s'implanter en ce lieu et s'y maintenir avec succès, assurer les fonctions médicales de Sarpédon.

Sur ce point, l'évolution de la tradition littéraire relative à Thècle est révélatrice. Dans les plus anciennes versions de la légende, la ville de Séleucie est à peine citée comme l'étape finale du parcours de la sainte, sans aucune référence à une activité quelconque de Thècle en ce lieu. Les manuscrits $\mathrm{E}, \mathrm{F}$, $\mathrm{K}$ et $\mathrm{L}$ des Actes de Paul et de Thècle s'achèvent sur les mots : « [...] elle partit pour Séleucie; et, ayant éclairé beaucoup de gens par la parole de Dieu, elle s'endormit d'un beau sommeil ${ }^{83}$. Mais, dès que l'existence de la martyre dans la cité cilicienne est décrite plus longuement, Thècle est présentée comme une guérisseuse : «[...] et des guérisons se produisirent par elle. Aussi toute la ville et ses environs, l'ayant appris, lui apportaient leurs malades sur la montagne; et, avant même qu'ils s'approchent de la porte, aussitôt ils étaient guéris, de quelque maladie qu'ils fussent attaqués; et les esprits impurs sortaient en vociférant; et tous recouvraient la santé de tout leur corps, $[\ldots] »^{84}$. Ces compétences médicales semblent donc bien résulter de l'installation de Thècle à Séleucie. Intrinsèquement, la martyre ne possédait aucune qualification thérapeutique. Cet aspect de sa personnalité, aujourd'hui prépondérant - Thècle est généralement présentée comme une sainte guérisseuse - s'est développé lors de l'implantation de son culte à Séleucie du Calycadnos. Plus encore, la Vie.et les Miracles démontrent qu'au v ${ }^{e}$ siècle ap.

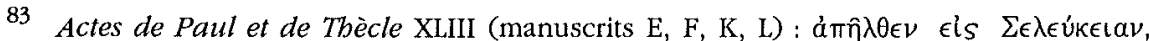

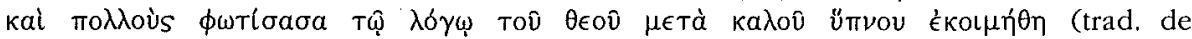
Vouaux, 1913).

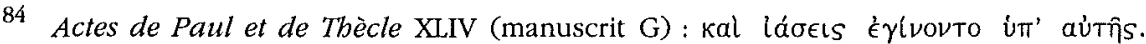

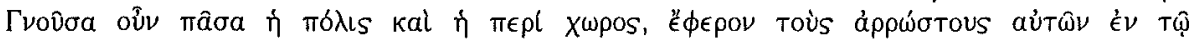

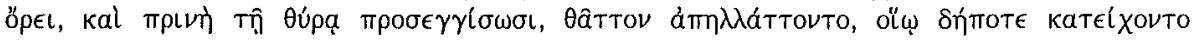

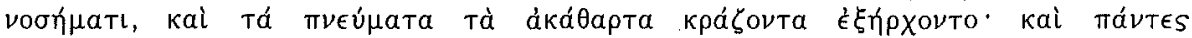

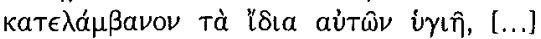


J.-C., ses fonctions médicales s'étaient imposées au point que son sanctuaire séleucien était devenu « un lieu public de cure ${ }^{85}$.

Si elle a revêtu ce rôle de guérisseuse à Séleucie, c'est qu'elle devait s'y mesurer à un adversaire précisément réputé et vénéré pour son savoir médical. Le culte de ce rival païen était profondément ancré dans la vie religieuse des habitants. Il avait déjà subi, au cours de sa longue histoire, une évolution fondamentale: Sarpédon, le propriétaire originel, avait été remplacé, ou en tout cas confondu avec Apollon. Les fidèles s'adressaient, depuis lors, à Apollon Sarpédonios, ou même toujours à Sarpédon. Pour s'imposer, la martyre ne s'est pas identifiée à l'ancien patron du culte. Au contraire, elle s'y est opposée avec force; son premier souci, dans les Miracles, est de rendre muet l'oracle sarpédonien ${ }^{86}$. Elle a néanmoins été contrainte d'endosser certaines fonctions auparavant dévolues à Sarpédon, en particulier ses interventions thérapeutiques. Thècle n'est donc pas, à mes yeux, une forme de Sarpédon ou d'Apollon Sarpédonios chrétien. Son culte ne peut être réduit au produit de la christianisation des pratiques païennes. A l'inverse, l'Église a choisi, afin de lutter efficacement contre ses concurrents païens, une personnalité distincte, tout à fait originale. Figure typique du christianisme, Thècle s'est efforcée de proposer aux fidèles un modèle spirituel différent, nourri des conceptions chrétiennes. Mais, pour répondre aux attentes des fidèles, elle a été dotée de qualités empruntées à son prédécesseur. Elle a ainsi acquis un rôle similaire de médecin, indispensable à son acceptation par les indigènes.

Séleucie constitue donc un cas manifeste de continuité de culte entre les époques païenne et chrétienne. Thècle y succède à Apollon Sarpédonios, qui s'était lui-même substitué à Sarpédon. Les cultes de Thècle et de Sarpédon partagent plusieurs particularités ${ }^{87}$, qui ont vraisemblablement soutenu leur rapprochement ${ }^{88}$. L'un comme l'autre avaient pour cadre un sanctuaire extraurbain, établi dans un endroit isolé, à quelque distance de la cité. La localisation exacte de l'oracle sarpédonien, installé sur un promontoire au bord de la $\operatorname{mer}^{89}$, reste à ce jour inconnue. Les témoignages littéraires laissent supposer qu'il occupait le cap Sarpédon, sur la côté méridionale de Séleucie, à proximité de la tombe de Sarpédon ${ }^{90}$. Quant au sanctuaire de Thècle, il

\footnotetext{
85 Vie de sainte Thècle XXVIII, 11-15.

86 Miracles de sainte Thècle I, 15-20.

87 Cf. Dagron, o.c. (n. 35), p. 87-88.
}

88 Précédemment, certaines études avaient conclu que Thècle avait succédé non à Sarpédon, mais à Athéna. Le culte de cette dernière est effectivement attesté à Séleucie sur l'acropole au centre de la ville (Vie de sainte Thècle XXVII, 56-62; Miracles de sainte Thècle II). Il s'agissait donc du culte protecteur de la cité dont Athéna était la déesse majeure. Ce culte n'est donc pas comparable à celui de Thècle, centre extra-urbain de pèlerinage.

89 Cf. Vie de sainte Thècle XXVII, 53-54; Miracles de sainte Thècle I, 9-10.

90 Supra, p. 114-115. 
s'élevait sur une colline au sud de Séleucie ${ }^{91}$. Les vestiges de cet imposant centre cultuel ont été mis au jour près de l'actuel village de Becili ${ }^{92}$, dont le nom primitif d'Ayatekla trahit la dévotion voisine à Thècle. En plus des indications fournies par les textes, notre connaissance de l'occupation du site repose sur les résultats des fouilles archéologiques entreprises dès $1907^{93}$. Les édifices sacrés étaient protégés par un puissant mur d'enceinte muni de tours et de portes. Outre de nombreuses citernes et des bains, ce péribole comprenait, à la fin du $\mathrm{v}^{\mathrm{e}}$ siècle ap. J.-C., une grotte ainsi que trois églises dont la plus méridionale avait été bâtie au-dessus de la grotte. Cependant, la construction tardive de ces monuments - les trois églises ont été édifiées dans la seconde moitié $d u \mathrm{~V}^{\mathrm{e}}$ siècle ap. J.-C. au plus tôt - interdit leur identification au martyrium primitif, siège du plus ancien culte en l'honneur de Thècle. Même si la géographie des cultes de Sarpédon et de Thècle comporte encore d'importantes zones d'ombre, il est certain que leurs sanctuaires respectifs n'ont pas été fondés sur le même site ${ }^{94}$. Ils occupaient, néanmoins, des emplacements similaires, des lieux isolés en dehors de la ville et organisés autour d'un tombeau. Ces sites retirés et déserts se prêtaient particulièrement bien au développement de cultes oraculaires et guérisseurs, dont la renommée dépassait largement le cadre de la ville de Séleucie. De tels centres de pèlerinage accueillaient de nombreux fidèles originaires de toute la région.

Outre la nature et la localisation de leur culte, Sarpédon et Thècle avaient en commun des légendes étonnamment semblables dans leur déroulement. L'une comme l'autre sont le fruit d'une élaboration littéraire très intense qui a donné corps et vie à des personnages fictifs, ou dont l'historicité est contestable à de nombreux égards ${ }^{95}$. Le résultat nous présente deux étrangers, un

91 Le sanctuaire de Thècle se dressait sur une hauteur proche de Séleucie, là où la sainte avait achevé son existence. Cf. Vie de sainte Thècle XXVII, 49-52.

92 La dénomination de ce site a fréquemment changé au cours de l'histoire : outre les appellations de Becili et d'Ayatekla, il a également porté le nom de Meriamlik.

93 Les fouilles de la cité-sainte de Thècle à Becili ont été publiées dans E. Herzfeld et S. GuYer, Monumenta Asiae Minoris Antiqua, II (Meriamlik und Korykos, zwei christliche Ruinenstätten des Rauben Kilikiens), Manchester, 1930.

94 Non seulement la topographie des sites destinés aux cultes de Sarpédon et de Thècle interdit leur identification, mais, en plus, l'auteur des Miracles explique qu'après la victoire de Thècle sur Sarpédon, ce dernier a abandonné le site qu'il occupait; ce site, ajoute-t-il, a alors été récupéré par des moines (Miracles de sainte Thècle I, 20-23), preuve que la martyre ne s'y est pas installée. Notre hagiographe n'aurait, en effet, pas manqué d'indiquer un tel événement.

95 Une étude minutieuse de la manière dont les sources chrétiennes ont été utilisées par l'auteur des Actes de Paul montre que, s'il a respecté le cadre historique, il a transformé les épisodes qui y prenaient place. Thècle comme Paul est donc probablement une figure historique, une martyre du ${ }_{1}^{\text {er }}$ siècle de notre ère, originaire du sud-est de l'Asie Mineure, mais ses aventures sont certainement le produit de l'imagination de l'auteur. $C f$. Vouaux, o.c. (n. 54), p. 127-129. 
homme d'une part, une femme de l'autre ${ }^{96}$, échoués, après diverses péripéties, à Séleucie où ils trouvent la mort et sont enterrés; leur tombeau devient alors le siège d'un culte. Il s'agit donc de deux figures humaines élevées, au terme de leur existence terrestre, à un rang surhumain de dieu ou de sainte. Les quelques allusions au Sarpédon séleucien contenues dans la Vie et les Miracles de Thècle le désignent toujours comme un $\delta \alpha\lfloor\mu \omega \nu$, c'està-dire comme un dieu ${ }^{97}$. L'auteur chrétien réserve, en effet, la dénomination de $\delta a i ́ \mu \omega \nu$ aux divinités païennes, qu'il s'agisse d'Athéna, d'Aphrodite, de Zeus ou d'Apollon ${ }^{98}$. Il confère donc à Sarpédon un statut identique à celui des grandes divinités olympiennes. Quant au culte rendu à ce $\delta a l \mu \omega \nu$, les maigres indications apportées par le double récit hagiographique ne témoifgnent pas de pratiques rituelles particulières, propres à un culte hérö̈que ${ }^{99}$. Sarpédon était considéré et vénéré comme un dieu.

L'auteur des Miracles expose la légende de Sarpédon telle qu'elle était véhiculée en Cilicie. Puis il en propose une explication évhémériste : Sarpédon était un « homme [...], qui avait pris le nom d'un démon et gagné une réputation d'oracle et de devin, et qui, pour cette raison, passait pour être un dieu auprès des esprits faibles (car en s'écoulant le temps engendre bien des illusions de cette sorte, que les hommes acceptent sans critique, faisant des dieux à partir de fables) [...] ${ }^{100}$. Dans cette interprétation, Sarpédon est donc passé, aux yeux des autochtones, du statut d'homme (ávríp), à celui de dieu

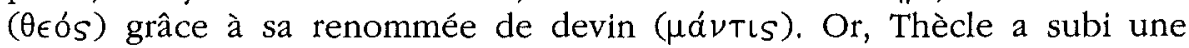
évolution très similaire: dans les Actes apocrypbes de Paul, elle est une

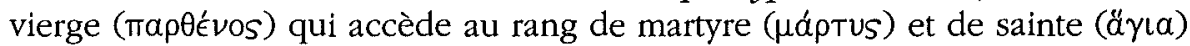
après avoir enduré diverses épreuves et réalisé plusieurs miracles.

En plus de la structure générale de la narration, les légendes de Sarpédon et de Thècle se rapprochent quant à la place accordée à Séleucie dans le récit. Le lien de la cité cilicienne avec la martyre est aussi lâche dans les

96 Sarpédon (Miracles de sainte Thècle I, 4) comme Thècle (idem II, 9) sont qualifiés de ḱévos par rapport à Séleucie. Cf. DaGron, o.c. (n. 35), p. 88.

97 Cf. Vie de sainte Thècle XXVII, 53-54. Miracles de sainte Thècle I, 10; XI, 13, 23, 36; $\mathrm{XL}, 30-31$.

98 Cf. Vie de sainte Thècle XXVII, 57. Miracles de sainte Thècle II, 4-5; IV, 2, 14, 18; XI, 30-31. - Sur les $\delta a l \mu o v \in S$ mentionnés dans la Vie et les Miracles de Thècle, voir Dagron, o.c. (n. 35$)$, p. $87-89$.

99 A. Verbanck-Piérard a montré que d'autres guérisseurs célèbres tels Asclépios ou Amphiaraos ne faisaient l'objet d'aucune pratique rituelle spécifique qui soutiendrait l'assimilation de leurs cultes à des cultes héroïques. Ils possédaient les mêmes fonctions, la même iconographie et le même culte que les dieux guérisseurs. $C f$. A. VerbancKPIÉrard, "Les héros guérisseurs : des dieux comme les autres! A propos des cultes médicaux dans l'Attique classique", Kernos, Suppl. 10 (2000), p. 281-332.

${ }^{100}$ Miracles de sainte Thècle I, 10-13 (trad. de Dagron, 1978) : Tov̂Tov [Tòv ăvôpa ...]

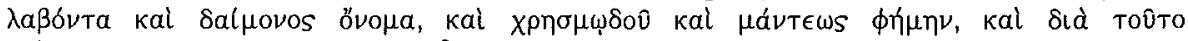

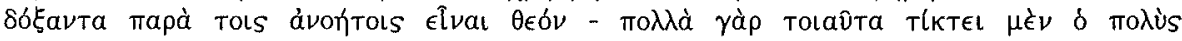

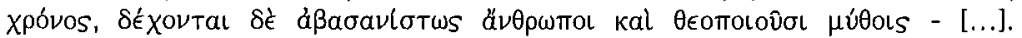


manuscrits les plus anciens des Actes de Paul qu'il ne l'est avec le fils de Zeus dans les versions ordinaires de la mythologie de Sarpédon. Après ses aventures à Iconium et Antioche de Pisidie, la sainte se retire à Séleucie ${ }^{101}$, tandis que le héros lycien vient en aide à son oncle, roi de la Cilicie voisine ${ }^{102}$. Par contre, au $\mathrm{v}^{\mathrm{e}}$ siècle ap. J.-C., dans la Vie et les Miracles, Thècle est présentée comme la sainte patronne de Séleucie alors que l'ancrage cilicien de Sarpédon est affirmé par une variante régionale de sa légende. Le rôle grandissant de Séleucie dans leurs légendes respectives trahit vraisemblablement la fixation tardive de leurs cultes dans cette cité. Le rayonnement acquis par la dévotion rendue à Sarpédon, puis à Thècle dans la ville cilicienne a nécessité une justification légendaire de ces cultes. Leur renommée grandissante a donc entraîné un vaste mouvement de reconstruction littéraire autour de leurs deux figures.

Enfin, l'examen des sources chrétiennes apporte également un indice supplémentaire en faveur de la thèse d'un culte conjoint d'Artémis et d'Apollon à Séleucie. Les Actes de Paul è de Thècle précisent, dans les manuscrits les plus prolixes sur l'existence menée par la sainte à Séleucie, qu'aux yeux des indigènes, Thècle tenait son pouvoir guérisseur de la déesse Artémis. "Ils [les médecins de la cité] disaient en effet que vierge, elle servait Artémis, et que c'était pour cette raison qu'elle avait la puissance de guérir. " ${ }^{103} \mathrm{Il}$ s'agit d'un écho chrétien du culte rendu à Séleucie en l'honneur de la déesse chasseresse. Ces écrits apocryphes pourraient donc confirmer le témoignage de Strabon. Plus encore, ils attribuent à Artémis des pouvoirs guérisseurs qu'elle aurait transmis à Thècle. Comme vierges, ces deux figures présentent des similitudes. Les manuscrits chrétiens affirment que la sainte servait Artémis, et qu'elle lui était même consacrée ${ }^{104}$; ils expriment ainsi un lien cultuel profond entre la déesse et la martyre. Thècle aurait été assimilée à Artémis à laquelle elle se serait progressivement substituée.

Cette conception semble s'opposer à la succession cultuelle retracée cidessus : Thècle aurait hérité ses facultés thérapeutiques d'Apollon, qui les tenait lui-même de Sarpédon. Ces deux hypothèses ne sont cependant pas incompatibles : Thècle a succédé, non pas à Apollon, non pas à Artémis, mais à Apollon et Artémis Sarpédoniens. Les jumeaux divins ont pris la place du $\delta a\{\mu \omega v$ local, dont le souvenir est d'ailleurs demeuré très vivace dans les

101 Actes de Paul et de Thècle XLIII (manuscrits E, F, K, L).

102 APollod, III, 1, 2.

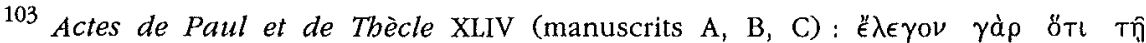

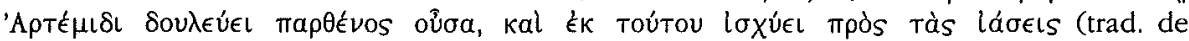
Vouaux, 1913). - Les manuscrits $G$ et $M$ affirment également que Thècle était consacrée à Artémis et que ses dons de thérapeute lui venaient de la sœur d'Apollon : Aütn

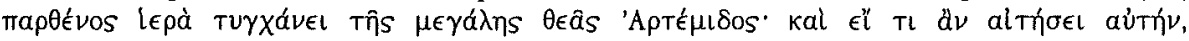

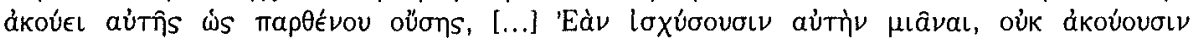

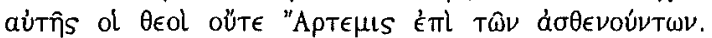

104 Ibid. 
croyances des autochtones; ces derniers se sont adressés à leur protecteur ancestral jusqu'en pleine époque chrétienne. La sainte qui avait alors pris le contrôle du culte possédait les qualités propres à assurer le succès de son implantation. Thècle partageait les compétences divinatoires, notamment médicales d'Apollon Sarpédonios. Mais, par sa nature de femme et de vierge, elle était plus proche d'Artémis, dont les Actes apocrypbes indiquent, de plus, le pouvoir de guérison. Si le culte de Thècle a acquis une telle renommée à Séleucie, si son sanctuaire a connu un développement architectural aussi important, c'est précisément parce qu'il remplaçait remarquablement la dévotion païenne antérieure, à savoir un culte commun d'Apollon et d'Artémis Sarpédoniens, un culte à vocation médicale.

Il reste toutefois une dernière objection à lever. Si le culte extra-urbain dépendant de Séleucie, dévolu à Sarpédon, puis à Apollon et Artémis, et enfin à Thècle, était un culte thérapeutique, pourquoi les deux seules consultations mentionnées dans la littérature païenne (chez Diodore et Zosime) ne concernent-elles pas des problèmes de santé ? Probablement parce que, d'une part, cet oracle, comme la plupart des sanctuaires médicaux de l'Antiquité ${ }^{105}$, n'accordait pas exclusivement des révélations de nature médicale. Des questions d'ordre politique, un des domaines les plus importants aux yeux des Anciens, pouvaient aussi être adressées à ces dieux réputés pour leurs facultés divinatoires. D'autre part, parce que les guérisons miraculeuses dépendaient de la sphère privée et ne présentaient, dès lors, aucun intérêt pour les historiens. Il s'agissait de miracles individuels, dont on ne possède, par ailleurs, que très peu de récits dans l'ensemble des temenoi thérapeutiques antiques ${ }^{106}$.

$*$

Au vu des similitudes présentées par les cultes séleuciens de Sarpédon et de Thècle, il me semble assuré que la martyre chrétienne a dû prendre la place du $\delta a i ́ \mu \omega \nu$ païen au terme d'une concurrence assez rude. Même localisation du culte dans un site extra-urbain; même type de sanctuaire, siège d'un pèlerinage organisé autour d'un tombeau; même récit légendaire mettant en scène une figure humaine étrangère à la cité, élevée au rang d'être

105 Asclépios lui-même, pourtant dieu de la médecine par excellence, est intervenu, à Epidaure, au profit de fidèles préoccupés par des problèmes sans aucune implication médicale. Un des miracles épidaurien $\left(I G \mathrm{IV}^{2}, 1,121,79-89\right)$ concerne ainsi la réparation d'une coupe brisée.

106 La majorité des récits de guérison (iamata) conservés proviennent des Asclepieia d'Épidaure ( $\left.I G I^{2} V^{2}, 1,121-124\right)$, de Lébéna, en Crète (Inscriptiones Creticae I, XVI, 9, 17, 18, 19, 24) et de Rome ( $C I G, 5980 ; I G$ XIV, 966). Gravés sur de grandes stèles de pierre exposées dans le sanctuaire, ils étaient destinés à affermir la foi des fidèles venus consulter le dieu. Pareilles guérisons ne sont que rarement rapportées dans des ouvrages officiels. La mention de consultations thérapeutiques est souvent justifiée par l'identité du bénéficiaire de la cure; ainsi Hérodien (IV, 8, 3) relate les visites de Caracalla dans le sanctuaire pergaménien. 
extraordinaire après un intense mouvement de reconstruction littéraire. Et surtout, même champ d'intervention, à savoir la révélation prophétique, en particulier la divination médicale exercée par ces deux guérisseurs renommés dans toute la région. Autant de points communs qui confirment la succession de Sarpédon à Thècle, via Apollon et Artémis Sarpédoniens, à la tête de ce culte de Séleucie. La pérennité cultuelle y fut telle que le rôle médical du sanctuaire s'est perpétué à toutes les époques, malgré les changements de destinataire. Plusieurs siècles avant l'arrivée de Thècle, Sarpédon était déjà consulté par des malades, comme il l'était encore alors même que sa rivale chrétienne était déjà installée dans la cité cilicienne. La vocation thérapeutique du culte explique d'ailleurs son maintien : le secours du patron du culte, dieu ou sainte, était indispensable aux fidèles touchés par la maladie.

Université de Liège

Cécile Nissen

Archéologie grecque

$1 \mathrm{~b}$, quai Roosevelt

B - 4000 LIÈGE 